\title{
Convective Generation of Equatorial Superrotation in Planetary Atmospheres
}

\author{
JUNJUN LIU AND TAPIO SCHNEIDER \\ California Institute of Technology, Pasadena, California
}

(Manuscript received 7 December 2010, in final form 6 June 2011)

\begin{abstract}
In rapidly rotating planetary atmospheres that are heated from below, equatorial superrotation can occur through convective generation of equatorial Rossby waves. If the heating from below is sufficiently strong that convection penetrates into the upper troposphere, then the convection generates equatorial Rossby waves, which can induce the equatorward angular momentum transport necessary for superrotation. This paper investigates the conditions under which the convective generation of equatorial Rossby waves and their angular momentum transport lead to superrotation. It also addresses how the strength and width of superrotating equatorial jets are controlled.

In simulations with an idealized general circulation model (GCM), the relative roles of baroclinicity, heating from below, and bottom drag are explored systematically. Equatorial superrotation generally occurs when the heating from below is sufficiently strong. However, the threshold heating at which the transition to superrotation occurs increases as the baroclinicity or the bottom drag increases. The greater the baroclinicity is, the stronger the angular momentum transport out of low latitudes by baroclinic eddies of extratropical origin. This competes with the angular momentum transport toward the equator by convectively generated Rossby waves and thus can inhibit a transition to superrotation. Equatorial bottom drag damps both the mean zonal flow and convectively generated Rossby waves, weakening the equatorward angular momentum transport as the drag increases; this can also inhibit a transition to superrotation. The strength of superrotating equatorial jets scales approximately with the square of their width. When they are sufficiently strong, their width, in turn, scales with the equatorial Rossby radius and thus depends on the thermal stratification of the equatorial atmosphere.

The results have broad implications for planetary atmospheres, particularly for how superrotation can be generated in giant planet atmospheres and in terrestrial atmospheres in warm climates.
\end{abstract}

\section{Introduction}

Superrotating (prograde) equatorial jets are ubiquitous in planetary atmospheres. Jupiter and Saturn exhibit equatorial superrotation, as do Venus and Titan (Porco et al. 2003; Sanchez-Lavega et al. 2007; Schubert 1983; Kostiuk et al. 2001). Yet it has remained unclear what distinguishes atmospheres that exhibit equatorial superrotation from those that do not. For example, in an order of magnitude sense, the giant planets Jupiter, Saturn, Uranus, and Neptune have similar atmospheres, with similar material properties, planetary rotation rates, and planetary radii. But Jupiter and Saturn have superrotating equatorial jets, whereas Uranus and Neptune have subrotating equatorial jets. Similarly, terrestrial atmospheres

Corresponding author address: Junjun Liu, California Institute of Technology, Mail Code 100-23, 1200 E. California Blvd., Pasadena, CA 91125.

E-mail: 1jj@gps.caltech.edu can become superrotating in warm climates (Caballero and Huber 2010), but the dynamical mechanisms that trigger the transition to superrotation are unclear.

We recently showed that randomly excited convection driven by sufficiently strong heating from below can lead to the generation of equatorial Rossby waves, which transport angular momentum toward the equator and can induce equatorial superrotation (Schneider and Liu 2009, hereafter SL09). The mechanisms involved are similar to those described by Suarez and Duffy (1992) and Saravanan (1993), who showed that a localized equatorial heat source can generate stationary Rossby waves and equatorial superrotation in terrestrial atmospheres. Our focus was on giant planet atmospheres with spatially uniform heating from below, which can generate transient Rossby waves and through them likewise equatorial superrotation. We proposed that the different strengths of the intrinsic heat fluxes relative to the differential radiative heating of the upper atmospheres are responsible for the existence of superrotation on Jupiter and Saturn

DOI: 10.1175/JAS-D-10-05013.1 
and subrotation on Uranus and Neptune (Liu and Schneider 2010, hereafter LS10). We showed that the different characteristics (strength, width) of the equatorial jets on these planets can be reproduced qualitatively in an energetically consistent general circulation model (GCM) with the respective planetary parameters.

Here we explore more systematically how heating from below, baroclinicity, and bottom drag determine when superrotation occurs. We use a GCM similar to the model of the upper atmospheres of the giant planets we used in SL09 and LS10, but with a planetary radius of only twice Earth's to reduce the computational cost of the simulations. We vary the strength of a heat flux imposed at the bottom boundary (which represents the intrinsic heat flux on giant planets); we vary the meridional gradient of the insolation imposed at the top of the atmosphere to vary the baroclinicity of the atmosphere; and we vary the bottom drag. All three factors have been shown previously to influence when superrotation occurs (SL09; LS10). For example, a stronger heat flux at the bottom boundary generally leads to a stronger convective Rossby wave source and so can enhance the angular momentum transport into the equatorial region, facilitating a transition to superrotation. Stronger baroclinicity leads to stronger baroclinic eddies of extratropical origin, which transport angular momentum out of the equatorial region, inhibiting a transition to superrotation (LS10). We investigate how the transition to superrotation occurs and the characteristics of the superrotating jets as the relative importance of these partially competing factors is varied.

Section 2 describes the GCM and the simulations conducted. Section 3 investigates the transition to superrotation as the heat flux at the bottom boundary strengthens. Section 4 discusses how the meridional insolation gradient influences the transition to superrotation. Section 5 investigates the effect of bottom drag. Section 6 discusses how the strength and width of equatorial superrotating jets are controlled. Section 7 discusses the conclusions, some open questions, and broader implications for planetary atmospheres.

\section{Model and simulations}

\section{a. General circulation model}

We use a variant of the three-dimensional GCM introduced in SL09 and LS10. The rotation rate, gravitational acceleration, and material properties of the atmosphere are those of Jupiter, but the planetary radius is twice Earth's. The GCM integrates the primitive equations for a dry ideal-gas atmosphere using the spectral transform method in the horizontal and finite differences in the vertical. The horizontal resolution is T85. The vertical

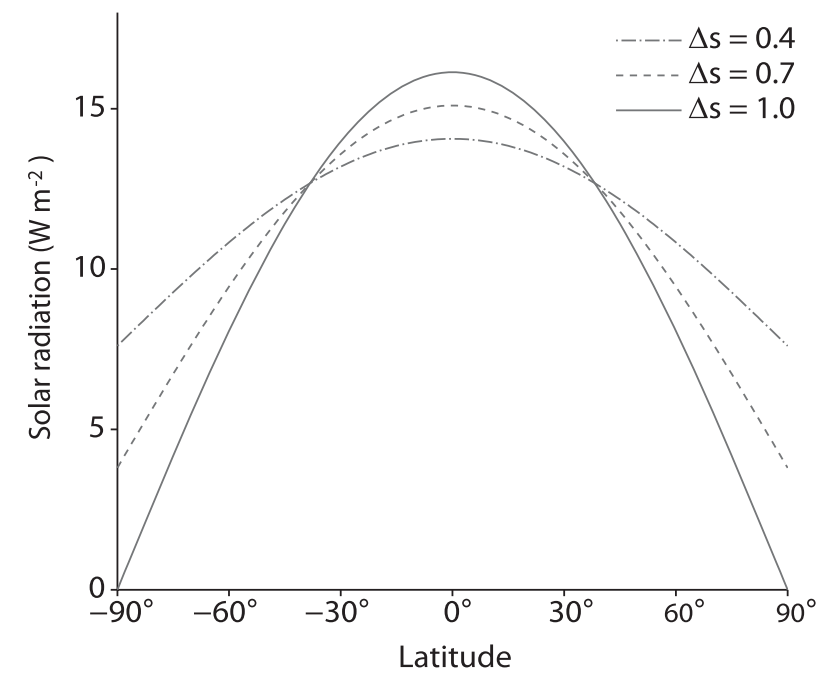

FIG. 1. Distribution of the solar radiation at the top of the atmosphere for different insolation gradients: $\Delta s=0.4$ (dasheddotted), $\Delta s=0.7$ (dashed), and $\Delta s=1.0$ (solid).

domain extends from the top of the atmosphere at zero pressure to a bottom boundary without topography and with a mean pressure of 3 bar. The vertical sigma coordinate is discretized with 30 evenly spaced levels.

Insolation at the top of the atmosphere is imposed with no diurnal cycle:

$$
S_{\mathrm{TOA}}=S_{0}\left(\frac{1-\Delta s}{4}+\frac{\Delta s}{\pi} \cos \phi\right)
$$

where $\phi$ is latitude and the artificial parameter $\Delta s$ controls the meridional insolation gradient. Varying the parameter $\Delta s$ varies the insolation gradient without changing the global mean:

$$
\int_{-\pi / 2}^{\pi / 2} S_{\mathrm{TOA}} \cos (\phi) d \phi=\frac{S_{0}}{4},
$$

where $S_{0}=50.7 \mathrm{~W} \mathrm{~m}^{-2}$ is Jupiter's solar constant (Lodders and Fegley 1998). Figure 1 shows $S_{\mathrm{TOA}}$ as a function of latitude for the different values of $\Delta s$ we use.

Radiative transfer in the atmosphere is treated as that in a semi-infinite atmosphere with wavelength-independent (gray) scattering and absorption of solar radiation and absorption of thermal radiation, using the two-stream approximation. This is an idealized representation of radiative transfer in gas giant atmospheres (SL09; LS10). The solar optical depth is $\tau_{s}=\tau_{s 0}\left(p / p_{0}\right)$, which is linear in pressure $p$ to represent scattering and absorption by a well-mixed absorber. We chose $\tau_{s 0}=3.0$ at $p_{0}=3$ bar, which gives a solar radiative flux qualitatively consistent with measurements by the Galileo probe inside Jupiter's atmosphere (Sromovsky et al. 1998). Thermal radiative 
transfer is calculated similarly, with a thermal optical depth $\tau_{l}=\tau_{l 0}\left(p / p_{0}\right)^{2}$ that is quadratic in pressure to represent collision-induced absorption of thermal radiation. We chose $\tau_{l 0}=80.0$, again as a rough approximation of thermal radiative transfer on Jupiter.

A spatially uniform and temporally constant intrinsic heat flux $F$ is imposed at the bottom boundary of the GCM. A quasi-equilibrium dry convection scheme is used to represent convection (Schneider and Walker 2006). It relaxes temperature profiles toward a convective profile with adiabatic lapse rate $\Gamma=g / c_{p}$ on a time scale of $6 \mathrm{~h}$. The appendix discusses the sensitivity of our results to aspects of the convection scheme.

The flow close to the bottom boundary is damped by linear (Rayleigh) drag. In applications to giant planet atmospheres, this drag can be thought of representing the magneto-hydrodynamic (MHD) drag acting in the electrically conducting planetary interior (SL09; LS10). As in Held and Suarez (1994), the Rayleigh drag coefficient $k(\phi, \sigma)$ decreases linearly in $\sigma$ from its value $k_{0}(\phi)$ at the bottom boundary $(\sigma=1)$ to zero at $\sigma_{b}=0.8$ (2.4 bar):

$$
k(\phi, \sigma)=k_{0}(\phi) \max \left(0, \frac{\sigma-\sigma_{b}}{1-\sigma_{b}}\right) .
$$

Above $\sigma_{b}$, there is no explicit friction, except subgrid-scale dissipation. We vary the magnitude and the dependence of the drag coefficient $k_{0}(\phi)$ on latitude to investigate how equatorial superrotation depends on drag.

Subgrid-scale dissipation (hyperdiffusion) is represented by an exponential cutoff filter in the vorticity, divergence, and temperature equations, with a cutoff wavenumber of 30 and a damping time scale of $0.5 \mathrm{~h}$ at the smallest resolved scale in all simulations (Smith et al. 2002). There is no damping for spherical wavenumbers less than 30 . We use the same subgrid-scale dissipation in all simulations to ensure that differences among them are not due to numerics. This is important because quantitative aspects of the superrotation in particular in simulations without bottom drag in the equatorial region are sensitive to the subgrid-scale dissipation. See SL09 for more model details.

\section{b. Simulations}

We conducted series of simulations varying the intrinsic heat flux at the bottom boundary, the meridional insolation gradient, and the bottom drag. The intrinsic heat flux $F$ was set to values ranging from 0 to $35.0 \mathrm{~W} \mathrm{~m}^{-2}$. The parameter $\Delta s$ controlling the insolation gradient was set to the values 0.4, 0.7, and 1.0 (Fig. 1). Three meridional dependencies of the drag coefficient were explored: Case $\mathrm{A}-$ no drag in the equatorial region (up to $33^{\circ}$ latitude) and constant drag coefficient $k_{0}=$ $10^{-2}$ day $^{-1}$ in higher latitudes; case B-constant drag coefficient with respect to latitude with $k_{0}=10^{-2}$ day $^{-1}$; and case $\mathrm{C}$-constant drag coefficient with respect to latitude with $k_{0}=5 \times 10^{-2}$ day $^{-1}$ (with 1 day $=86400 \mathrm{~s}$ ). Case A is a representation of the effect of MHD drag deep in a planetary atmosphere (at $\sim 0.84$ planetary radii) on the flow in the upper atmosphere; see SL09 and LS10 for a justification.

The time step is $300 \mathrm{~s}$ in simulations with imposed heat flux of $35.0 \mathrm{~W} \mathrm{~m}^{-2}$ for $\Delta s=0.4$ and $\Delta s=0.7$ and $450 \mathrm{~s}$ is in all other simulations. All simulations were integrated at least 20000 days, to ensure a statistically steady state has been reached; results shown are averages over at least 500 subsequent days.

\section{Variation of intrinsic heat flux}

Giant planet atmospheres are heated from above by scattering and absorption of solar radiation and from below by an intrinsic heat flux emanating from the interior. If the intrinsic heat flux is sufficiently weak, the atmosphere is usually stably stratified. As the intrinsic heat flux strengthens, the static stability of the atmosphere decreases, until lower layers become statically unstable and convection sets in. Once convection sets in, it can generate Rossby waves in the equatorial region, in particular near the convective outflows in the vicinity of the tropopause. Then, convectively generated Rossby waves that dissipate outside the equatorial region transport angular momentum into the equatorial region, thus potentially inducing equatorial superrotation (SL09).

The reason why convection can generate Rossby waves in the equatorial region is that there (where the Rossby number is order 1 and the Froude number is small) largescale horizontal temperature gradients are constrained to be weak, so the dominant thermodynamic balance is approximately (Charney 1963; Sobel et al. 2001)

$$
\nabla_{h} \cdot \boldsymbol{v}_{\chi} \approx \partial_{p}(Q / S)
$$

Here, $\boldsymbol{v}_{\chi}$ denotes the divergent horizontal flow component, $Q \stackrel{\chi}{=} D \theta / D t$ the diabatic heating rate, and $S=-\partial_{p} \theta$ the static stability with potential temperature $\theta$; the subscript $h$ on the differential operator $\nabla_{h}$ signifies the horizontal derivative. Thus, diabatic heating fluctuations, for example, in convection, lead to fluctuations in the horizontal divergence (4), particularly where the static stability $S$ changes rapidly with height (e.g., in the layer of convective outflows in the vicinity of the tropopause). Horizontal divergence fluctuations, in turn, are a source of equatorial Rossby waves; the fluctuating source of rotational flow (vorticity) 


$$
R^{\prime}=R-\bar{R}
$$

with

$$
R=-\zeta_{a}\left(\nabla_{h} \cdot \boldsymbol{v}_{\chi}\right)-\left(\boldsymbol{v}_{\chi} \cdot \nabla_{h}\right) \zeta_{a}
$$

can be taken to be the Rossby wave source (Sardeshmukh and Hoskins 1988). (The overbar denotes the isobaric zonal and temporal mean and primes deviations therefrom.) This indicates that rotational flow and so Rossby waves can be generated by stretching of absolute vorticity or advection of absolute vorticity by the divergent flow. Thus, the Rossby wave source $R^{\prime}$ can be expected to strengthen as the intrinsic heat flux and with it convective heating fluctuations and divergent flow fluctuations strengthen; (4) and (5) suggest it strengthens approximately linearly with the heat flux when the static stability $S$ does not vary strongly. The Rossby wave source can be expected to have largest magnitude in the upper troposphere, in the region of the convective outflows, because there convective heating fluctuations are large and so are vertical variations of the static stability, implying large divergence fluctuations (4). See SL09 and LS10 for a more detailed theoretical discussion.

Equatorial superrotation results through the convectively generated Rossby waves if their equatorward angular momentum transport exceeds the angular momentum transport out of low latitudes by the mean meridional circulation and/or by baroclinic eddies of extratropical origin. The transition to superrotation as the intrinsic heat flux strengthens is illustrated by the series of simulations with varying intrinsic heat flux at the bottom boundary. With weak insolation gradient $(\Delta s=$ $0.4)$ and with no bottom drag in the equatorial region and with a constant drag coefficient outside of it (case A in section $2 b$ ), superrotation occurs when the heat flux exceeds $\sim 15 \mathrm{~W} \mathrm{~m}^{-2}$ (Fig. 2, left column). The superrotating equatorial jet becomes stronger and wider as the intrinsic heat flux strengthens further.

That the waves responsible for the generation of equatorial superrotation are indeed Rossby waves can be seen in Fig. 3. The correlation coefficient between the streamfunction of the rotational horizontal flow and zonal flow fluctuations at an equatorial reference point shows the typical lobe structure of equatorial Rossby wave modes, with reflection symmetry of the horizontal flow around the equator; the meridional extent of the lobes is similar to the width of the superrotating jet (Fig. 3a). Embedded in the large-scale lobe structure are smaller-scale waves. Their phase lines tilt retrogradely away from the equator, indicating equatorward angular momentum transport (Peixoto and Oort 1992, ch. 11). The large-scale envelopes appear to represent packets of embedded smaller-scale
Rossby waves. The correlation coefficient between the horizontal divergence field and equatorial zonal flow fluctuations shows that the smaller-scale Rossby waves organize the divergence and thus the convection (Fig. 3b). A Hovmöller diagram of the horizontal streamfunction fluctuations shows that the wave packets propagate westward at $23 \mathrm{~m} \mathrm{~s}^{-1}$, consistent with them being Rossby wave packets (Fig. 3c). (See www.gps.caltech.edu/ tapio/ animations.html for animations of similar flow structures in the simulations of SL09 and LS10.)

In these simulations with relatively weak baroclinicity and no drag in the equatorial region, the transition to superrotation occurs soon after convection sets in. From the buoyancy frequency (Fig. 2, colors in right column), it is evident that convection sets in and first leads to an approximately neutrally stratified middle troposphere for an intrinsic heat flux $\geq 5.0 \mathrm{~W} \mathrm{~m}^{-2}$. For weaker heat fluxes, the atmosphere is stably stratified, and the equatorial flow is retrograde. The retrograde equatorial flow actually strengthens as the intrinsic heat flux increases from 0 to $5.0 \mathrm{~W} \mathrm{~m}^{-2}$ (Fig. 2, left column), apparently because the weakened static stability of the troposphere and the implied greater baroclinicity lead to more effective angular momentum transport out of low latitudes by extratropical baroclinic eddies. [The emergence of lowlatitude retrograde flow, on which the energy-containing baroclinic eddies cannot propagate (Charney 1969), self-limits baroclinic eddy transports out of low latitudes.]

As the heat flux strengthens beyond the threshold at which superrotation occurs, the superrotating equatorial jet widens and strengthens (Fig. 2, left column). The rms Rossby wave source (5) in the equatorial region increases approximately linearly with the heat flux, implying increased Rossby wave generation and increased angular momentum transport into the equatorial region (SL09). Several wave-mean flow feedbacks act at the transition to superrotation, which could render the transition as a function of heat flux nonlinear and possibly abrupt (cf. Suarez and Duffy 1992). For example, the emergence of prograde equatorial flow facilitates the propagation of Rossby waves out of the equatorial region and reduces the dissipation of the energy-containing extratropical eddies, which otherwise break near their critical latitudes before reaching the equator, near where the zonal flow changes from prograde to retrograde (Randel and Held 1991). This implies reduced angular momentum transport by extratropical baroclinic eddies out of low latitudes and possibly enhanced angular momentum transport by equatorial waves into low latitudes, thus representing a positive feedback that may amplify the transition to superrotation (Saravanan 1993; Held 1999). Or, once there is net eddy angular momentum flux convergence in the 

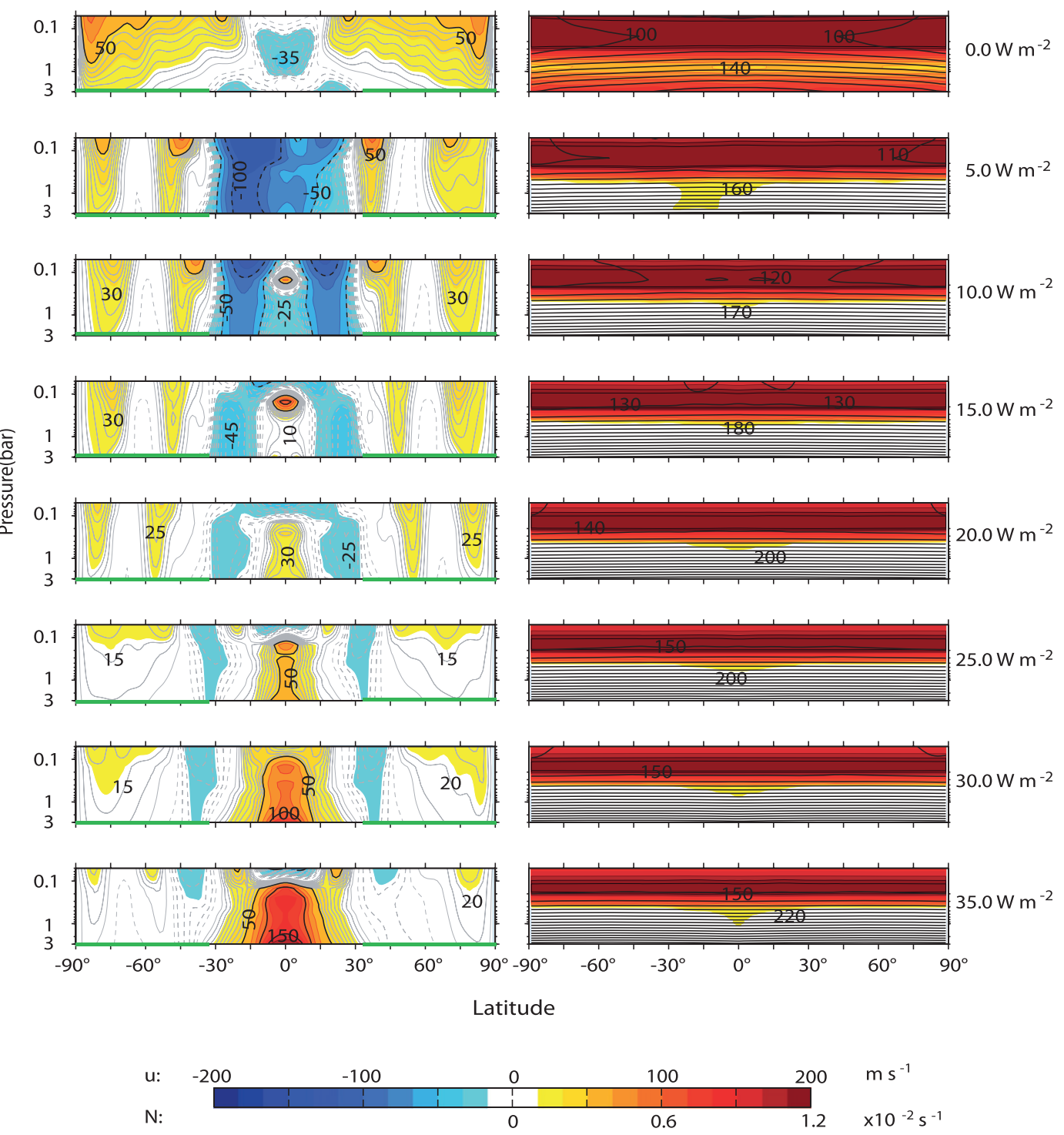

FIG. 2. Transition to equatorial superrotation as the intrinsic heat flux strengthens for weak insolation gradients $(\Delta s=$ 0.4 ) and no bottom drag in the equatorial region (case A). The intrinsic heat flux increases from (top) 0 to (bottom) $35 \mathrm{~W} \mathrm{~m}^{-2}$, taking the values indicated on the right. (left) Contours and colors show the zonal velocity, with red tones for prograde flow and blue tones for retrograde flow. Gray contours indicate zonal flow speeds between 5 and $45 \mathrm{~m} \mathrm{~s}^{-1}$, with contour interval $5 \mathrm{~m} \mathrm{~s}^{-1}$; black contours indicate zonal flow speeds of $50 \mathrm{~m} \mathrm{~s}^{-1}$ or greater, with contour interval $50 \mathrm{~m} \mathrm{~s}^{-1}$. (Black contours that would be inside gray contours are not shown.) (right) Contours show temperature, with contour interval $10 \mathrm{~K}$; colors show the buoyancy frequency $N$. The green part of the latitude axis marks the latitude with nonzero Rayleigh drag.

upper troposphere in low latitudes, the mean meridional circulation can reverse, because the dominant angular momentum balance in the upper branches of the mean meridional circulation is $(f+\bar{\zeta}) \bar{v} \approx S_{e}$, where $S_{e}$ is the eddy angular momentum flux divergence (e.g., Walker and Schneider 2006). Figure 4 shows an example of such a reversed mean meridional circulation. This implies convergence (rather than the usual divergence) of the mean meridional flow near the equator in the upper troposphere, where the Rossby wave source is strongest. Mean convergence $\left(\nabla_{h} \cdot \overline{\boldsymbol{v}}_{\chi}<0\right)$, in turn, implies that the first term in (5b) contains a contribution $-\left(\nabla_{h} \cdot \overline{\boldsymbol{v}}_{\chi}\right) \zeta^{\prime}$ that amplifies (rather than damps) vorticity fluctuations, representing an additional positive feedback that may amplify the transition to superrotation (SL09).

In our simulations, however, there appears to be a gradual transition to superrotation and a subsequent gradual strengthening of the prograde equatorial jet as 

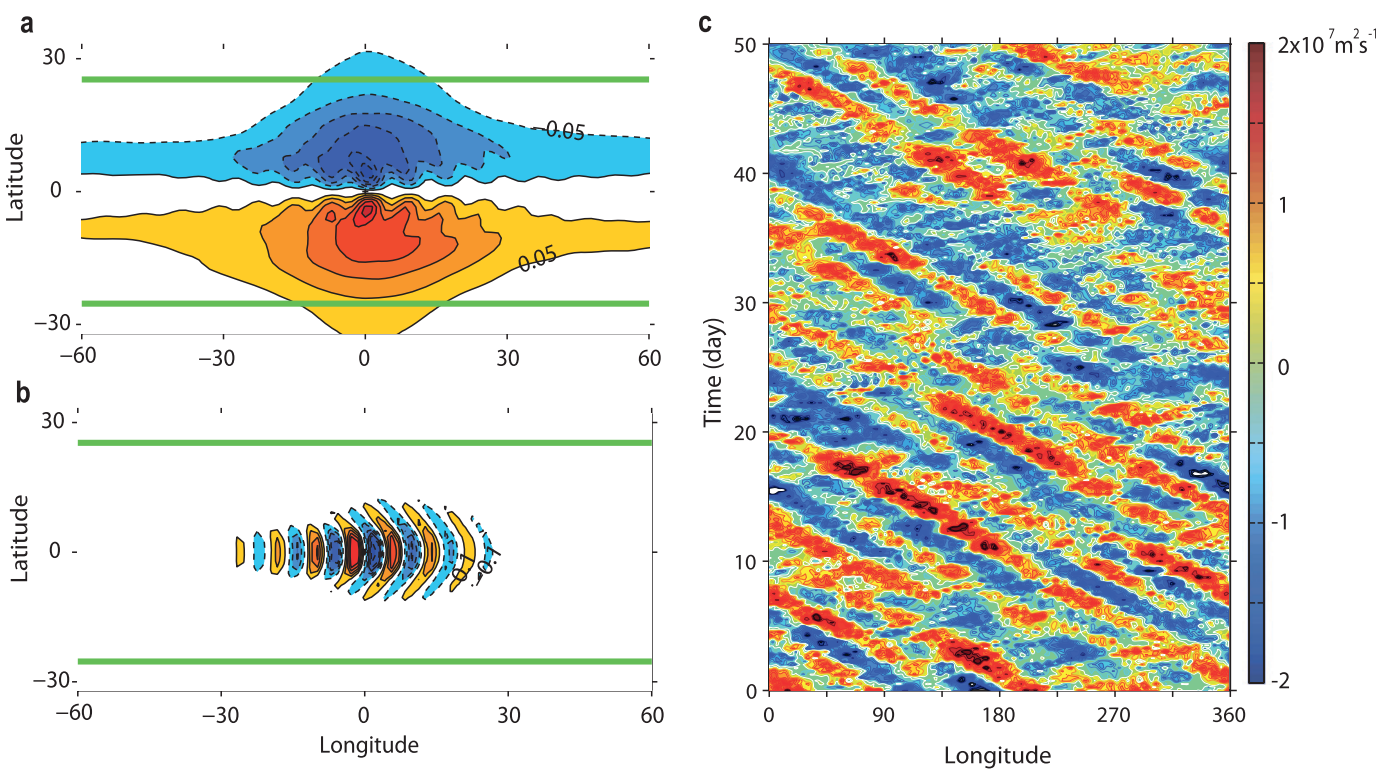

FIG. 3. Wave structures in the equatorial region at 0.85 bar for the simulation with intrinsic heat flux $30.0 \mathrm{~W} \mathrm{~m}^{-2}$, $\Delta s=0.4$, and no drag in the equatorial region. (a) Correlation coefficient between the streamfunction of the horizontal rotational flow and zonal flow fluctuations at a reference point on the equator $\left(0^{\circ}\right.$ longitude and $0^{\circ}$ latitude $)$. Solid contours (red tones) indicate positive correlation and dashed contours (blue tones) negative correlation, with contour levels from \pm 0.05 to \pm 0.4 separated by factors of 2 . The thick green lines in (a) [and (b)] show the latitudes of the zeros of the mean zonal wind and thus indicate the extent of the superrotating jet. (b) Correlation coefficient between the divergence of the horizontal flow and zonal flow fluctuations at the reference point. Solid contours (red tones) for positive correlation, and dashed contours (blue tones) for negative correlation, with contour levels from \pm 0.1 to \pm 0.8 separated by factors of 2 . (c) Hovmöller diagram of streamfunction fluctuations at $5^{\circ} \mathrm{N}$.

the intrinsic heat flux strengthens (Fig. 5a). Concomitantly, the width of the equatorial jet increases (Fig. 5b). In the simulations without bottom drag in the equatorial region, the strength and width of the equatorial jets is sensitive to the subgrid-scale dissipation, which represents the only dissipative process in low latitudes. For example, subgrid-scale dissipation that is less scale selective than the one we use and that damps larger scales more strongly generally weakens or inhibits equatorial superrotation. Nonetheless, the strength and width of the superrotating jets obey constraints, which we will discuss in section 6.

Away from the equator, the strengthening intrinsic heat flux and the decreasing tropospheric static stability it implies result in baroclinic eddy scales and off-equatorial jet widths that generally decrease with the heat flux (Fig. 2 ). Without a heat flux at the bottom boundary, there is a single broad jet in each hemisphere. As the heat flux strengthens, the static stability in the off-equatorial troposphere decreases, and so does the Rossby radius and the energy-containing eddy length scale, which scales approximately with the Rossby radius (Schneider 2006; Schneider and Walker 2008; SL09). The energy-containing eddy length scale, in turn, sets the scale of the off-equatorial jets, which thus likewise decreases. Additionally, the off-equatorial jets shift poleward as the superrotating equatorial jet widens. We will investigate the offequatorial dynamics in these simulations in greater detail elsewhere.

\section{Variation of meridional insolation gradient}

To investigate how baroclinicity influences when superrotation occurs, we conducted two series of simulations like those in section 3 but with stronger meridional insolation gradients: $\Delta s=0.7$ and 1.0 (versus $\Delta s=$ 0.4 in section 3 ). As the insolation gradient increases, the equator-to-pole temperature and potential temperature differences increase, both in radiative-convective equilibrium and in the dynamical equilibrium that establishes itself in the simulations. For example, for an intrinsic heat flux of $15.0 \mathrm{~W} \mathrm{~m}^{-2}$, the equator-to-pole potential temperature difference in radiative-convective equilibrium at a fixed pressure level in the convective layer increases from less than $10 \mathrm{~K}$ to more than $20 \mathrm{~K}$ as $\Delta s$ increases from 0.4 to 1.0 ; the analogous potential temperature difference in dynamical equilibrium increases from 3.5 to $6.0 \mathrm{~K}$ (Fig. 6). For this intrinsic heat flux, the atmosphere below the tropopause (at around 0.1 bar) is convectively unstable (e.g., Fig. 2, right column). Convection 

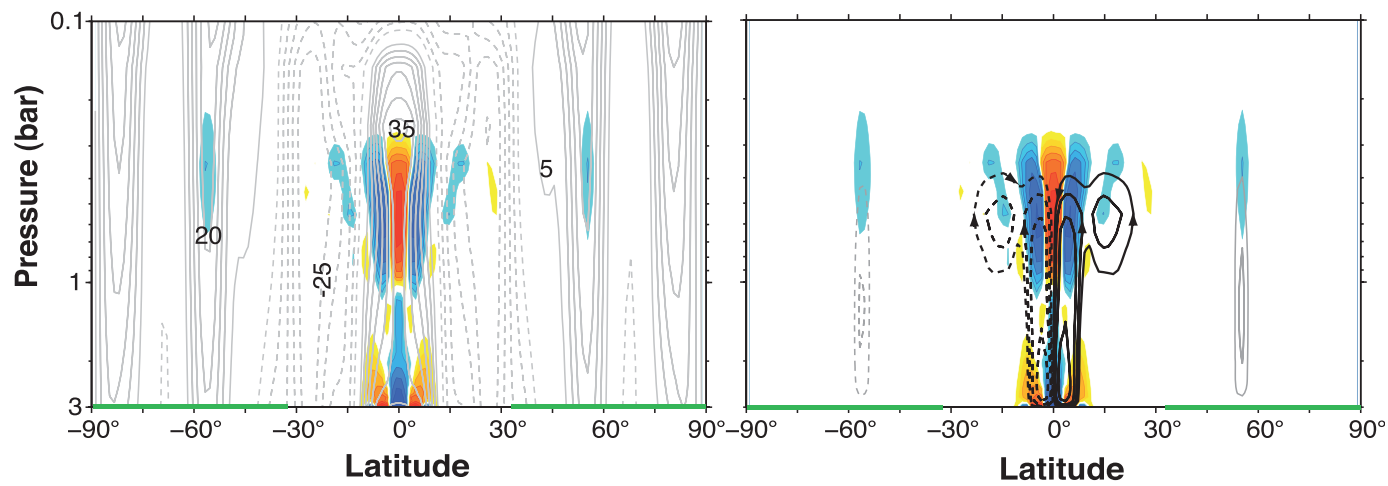

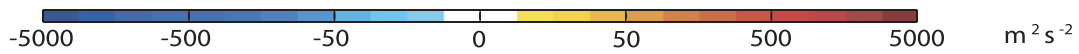

FIG. 4. Mean zonal velocity, mass flux streamfunction, and divergence $\operatorname{div}\left(\overline{u^{\prime} v^{\prime}} a \cos \phi\right)$ of meridional eddy angular momentum fluxes (color) in the simulation with an intrinsic heat flux of $20.0 \mathrm{~W} \mathrm{~m}^{-2}, \Delta s=0.4$, and no drag in the equatorial region. (left) Zonal-flow profiles (contours as in Fig. 2) and eddy momentum flux divergence (colors). (right) Mass flux streamfunction (contours) and the same eddy momentum flux divergence as at left (colors). The contouring for the streamfunction is logarithmic: gray contours from \pm 4 to $\pm 64 \times 10^{8} \mathrm{~kg} \mathrm{~s}^{-1}$; black contours for absolute values greater or equal to $\pm 128 \times 10^{8} \mathrm{~kg} \mathrm{~s}^{-1}$, with factor of 2 separating contour levels. (Gray contours that would be within black contours are not shown.) Solid contours indicate positive streamfunction values (counterclockwise rotation) and dashed contours negative streamfunction values (clockwise rotation). The contouring for the eddy momentum flux divergence is likewise logarithmic with the scale shown in the color bar. As in Fig. 2, the thick green parts of the latitude axes mark the latitudes with nonzero drag. Note that the mean meridional circulation near the equator closes in a bottom boundary layer, which forms although the boundary condition there is stress free.

approximately homogenizes entropy along angular momentum surfaces, so that the potential temperature away from the equator, where angular momentum surfaces are approximately vertical, is almost constant in the vertical (LS10). Hence, latitudinal potential temperature structures at different levels within the convective layer are essentially the same as those in Fig. 6.

As the baroclinicity increases with increasing insolation gradient, baroclinic eddy momentum fluxes strengthen, as expected from scaling theories of atmospheric macroturbulence (e.g., Schneider and Walker (2008). Baroclinic eddies transport angular momentum out of low latitudes into their midlatitude generation regions [see O'Gorman and Schneider (2008) for an illustration], so the angular momentum transport out of low latitudes strengthens, inhibiting a transition to superrotation (Saravanan 1993). As a result, everything else being equal, a stronger intrinsic heat flux is required for a transition to superrotation with larger insolation gradients.

Figure 7 shows how these expectations are borne out in the simulations with $\Delta s=1.0$. As in the simulations with $\Delta s=0.4$ (Fig. 2), convection first sets in and leads to an approximately neutrally stratified middle troposphere for an intrinsic heat flux $\geq 5.0 \mathrm{~W} \mathrm{~m}^{-2}$. But unlike in the simulations with $\Delta s=0.4$, the equatorial zonal flow for $\Delta s=1.0$ is subrotating in most of the column for intrinsic heat fluxes up to $35.0 \mathrm{~W} \mathrm{~m}^{-2}$. The magnitude of the subrotating flow decreases as the heat flux strengthens, and a localized prograde jet emerges in the equatorial upper troposphere, where the convective Rossby wave source is strongest-consistent with convective equatorial Rossby wave generation playing an increasingly important role in the angular momentum balance. But an outright transition to superrotation throughout the equatorial column does not occur for the heat fluxes we explored.

Away from the equator, the enhanced baroclinicity leads to stronger prograde jets, both because the increased meridional temperature gradients imply stronger prograde (thermal wind) shear of the jets and because the increased baroclinic angular momentum transport into midlatitudes implies a stronger zonal flow at the bottom boundary (cf. Figs. 3 and 7). (Angular momentum convergence in a vertical column is balanced by drag on the zonal flow at the bottom boundary.) The speed of the off-equatorial jets reaches $150 \mathrm{~m} \mathrm{~s}^{-1}$ in the upper atmosphere (Fig. 7).

The vertically averaged strength of the equatorial jets in all simulations with $\Delta s=0.4,0.7$, and 1.0 is shown in Fig. 5a. The simulations with $\Delta s=0.7$ lie between those with $\Delta s=0.4$ and 1.0 and exhibit a transition to superrotation for intrinsic heat fluxes $\geq 30 \mathrm{~W} \mathrm{~m}^{-2}$. The equatorial superrotating jets, however, are weaker and narrower than those in the simulations with the weaker insolation gradient with $\Delta s=0.4$ (Figs. 5a,b). 

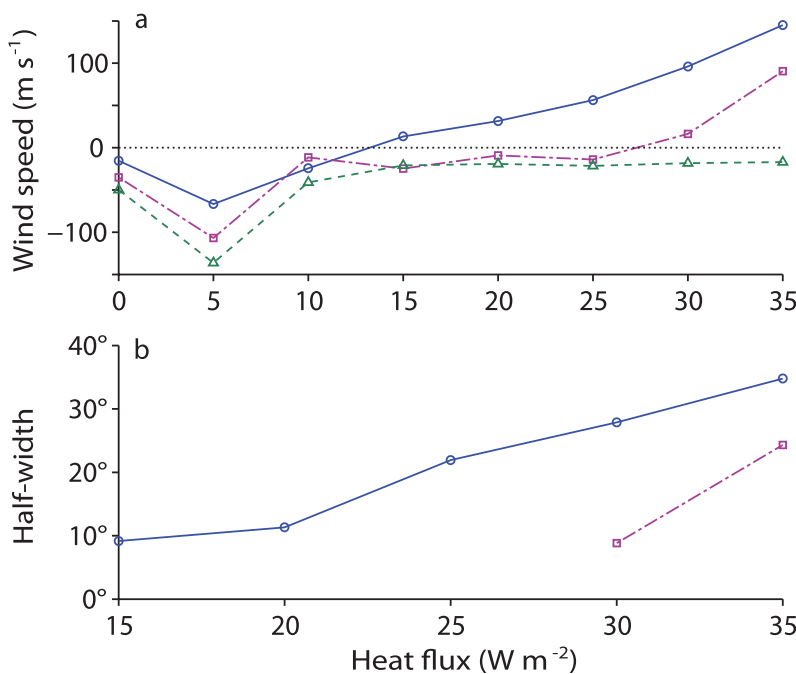

FIG. 5. Vertically averaged strength and width of the equatorial jets in the simulations with no bottom drag in the equatorial region (case A). (a) Equatorial zonal velocity as a function of the intrinsic heat flux, averaged from $-1^{\circ}$ to $1^{\circ}$ latitude meridionally and from $\sigma=$ 0.1 ( 0.3 bar) to $\sigma=0.8$ (2.4 bar) vertically. (b) Half-width of the equatorial jet (latitude of first zero of vertically averaged zonal velocity) as a function of the intrinsic heat flux. Blue circles with solid lines for $\Delta s=0.4$; magenta squares with dashed-dotted lines for $\Delta s=0.7$; green triangles with dashed lines for $\Delta s=1.0$.

\section{Variation of bottom drag}

To investigate how bottom drag influences when superrotation occurs, we conducted two series of simulations like those in section 3 with $\Delta s=0.4$ but with bottom drag in the equatorial region: with the same drag coefficient as outside the equatorial region in case A but now with the drag coefficient being constant at all latitudes (case B), and with a larger latitudinally constant drag coefficient (case C). When there is bottom drag in the equatorial region, the drag damps the zonal mean angular momentum directly and/or inhibits the meridional propagation of convectively generated waves. This can inhibit a transition to superrotation in at least two ways. First, bottom drag in the equatorial region damps the zonal flow near the bottom, which makes it possible for mean meridional circulations to bring air with low angular momentum from deeper in the atmosphere into the upper troposphere, thus inhibiting a transition to superrotation (Shell and Held 2004). Second, if instead of propagating meridionally out of their generation region, the convectively generated Rossby waves dissipate in the same latitude zone in which they are generated because of drag, they do not transport angular momentum meridionally (see, e.g., Held 2000 and SL09), likewise inhibiting a transition to superrotation.

Figure 8 shows the flow structures in the simulations with the weaker drag (case B). Comparison of Figs. 2

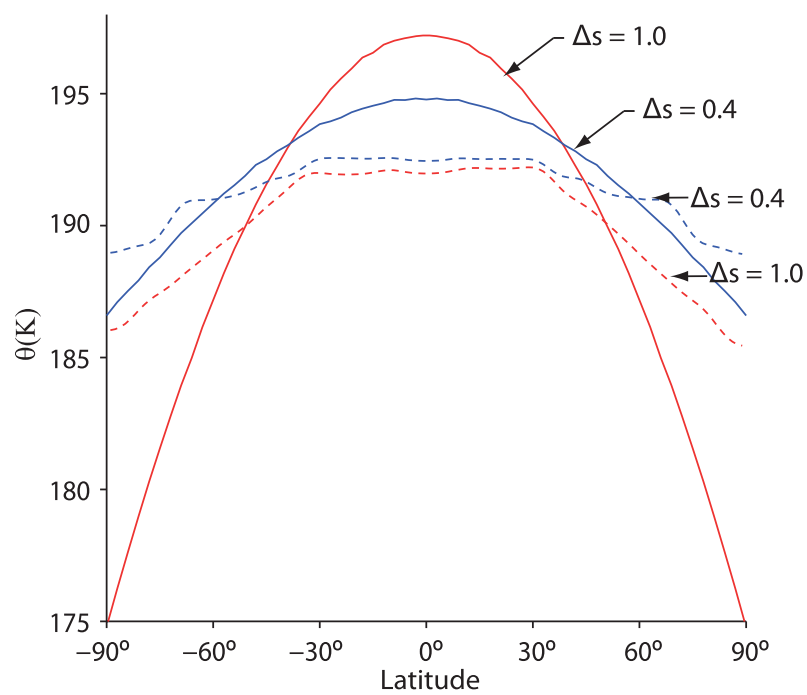

FIG. 6. Potential temperature at 2.0 bar in radiative-convective equilibrium (solid lines) and in dynamical equilibrium (dashed lines) for an intrinsic heat flux of $15.0 \mathrm{~W} \mathrm{~m}^{-2}$. Blue lines for a weak meridional insolation gradient $(\Delta s=0.4)$ and red lines for a strong meridional insolation gradient $(\Delta s=1.0)$.

and 8 shows that the thermal structures of the atmospheres are almost identical with or without drag in the equatorial region. In both sets of simulations, the atmosphere becomes neutrally stratified throughout much of the troposphere when the intrinsic heat flux reaches $5.0 \mathrm{~W} \mathrm{~m}^{-2}$. However, the stabilization of the thermal stratification in the equatorial troposphere for intrinsic heat fluxes greater than $5.0 \mathrm{~W} \mathrm{~m}^{-2}$, primarily produced by eddies, is weaker in the simulation with drag in the equatorial region, since the bottom drag weakens the convectively generated eddies.

Although the thermal structures of the atmospheres are similar with or without bottom drag in the equatorial region, the equatorial jets are weaker in the presence of drag in the equatorial region. For the case with drag in the equatorial region, the vertically averaged speed of the subrotating equatorial jet is reduced from $\sim 100$ to $\sim 20 \mathrm{~m} \mathrm{~s}^{-1}$ for the intrinsic heat flux of $5.0 \mathrm{~W} \mathrm{~m}^{-2}$. When the heat flux increases to $10.0 \mathrm{~W} \mathrm{~m}^{-2}$, a localized superrotating jet with a speed of $50.0 \mathrm{~m} \mathrm{~s}^{-1}$ emerges in the upper troposphere. Superrotation only occupies the entire troposphere when heat fluxes reach $15 \mathrm{~W} \mathrm{~m}^{-2}$. But even then the superrotating jet is weaker and narrower than in the case without bottom drag in the equatorial region (cf. Figs. 2 and 8). However, the structure of the off-equatorial flow is essentially unaffected by changing the bottom drag in the equatorial region.

As the bottom drag coefficient increases (case C), the strength and width of the equatorial superrotating jets are reduced even more (Fig. 9). The heat flux at which superrotation occupies the entire troposphere 

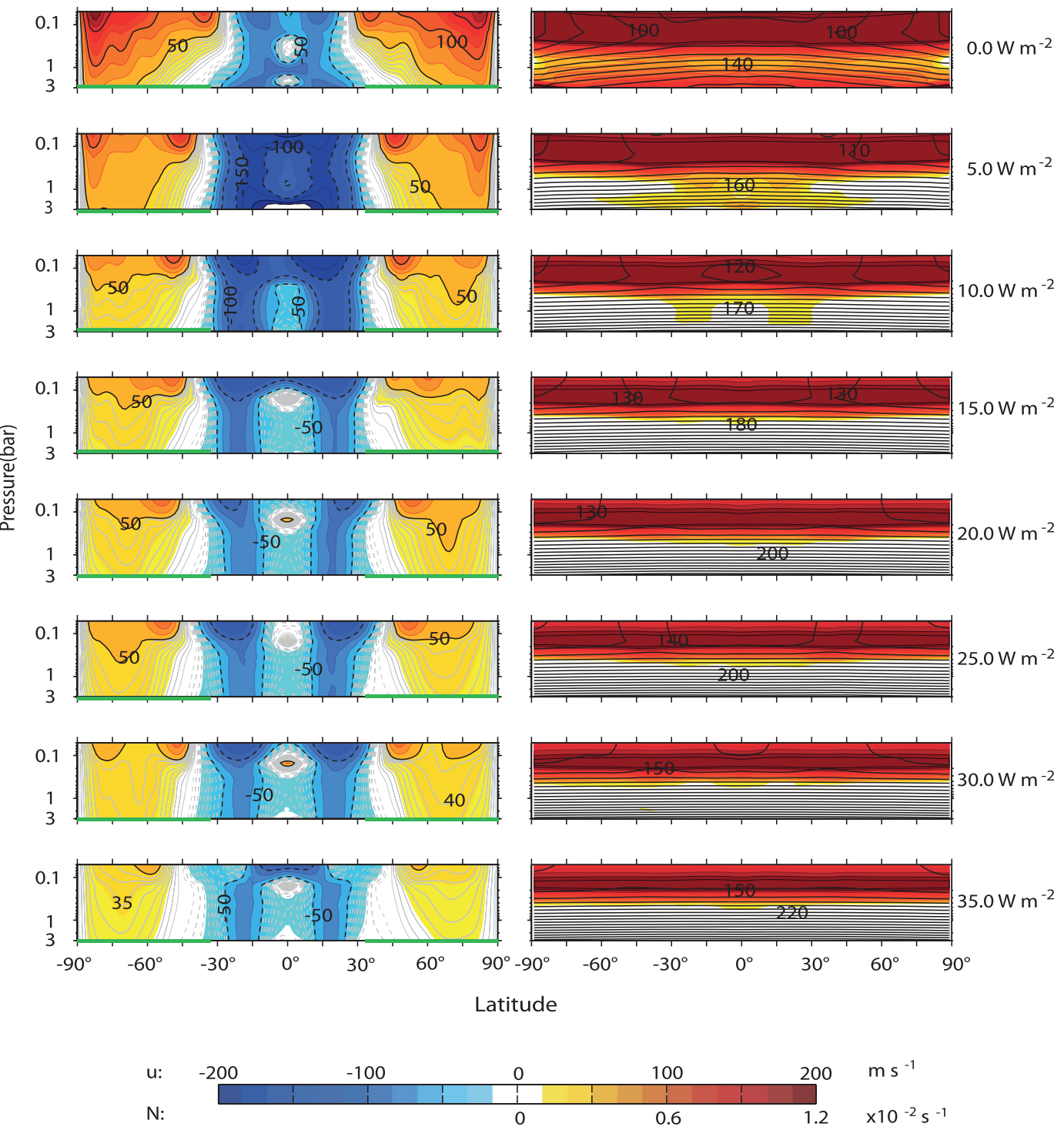

FIG. 7. Simulations with large meridional insolation gradient $\Delta s=1.0$ and no bottom drag in the equatorial region (case A). Contour intervals and plotting conventions as in Fig. 2.

is $\sim 20 \mathrm{~W} \mathrm{~m}^{-2}$, and the vertically averaged zonal velocity is smaller.

\section{Width and strength of superrotating jets \\ a. Theoretical constraints}

A vorticity homogenization argument gives an upper bound for the strength of an equatorial superrotating jet as a function of its width (Rhines 1994; SL09). The convective generation and dissipation of equatorial Rossby waves leads to the mixing of absolute vorticity (or potential vorticity, but potential vorticity in the presence of neutral stratification is not defined, and the distinction between the two is immaterial here because horizontal temperature gradients in the equatorial region are weak). The vorticity mixing reaches an end state and meridional Rossby wave propagation ceases when absolute vorticity is homogenized in each hemisphere. This end state is sketched in Fig. 10 (dashed magenta line). It has a barotropically stable vorticity jump at the equator, implying that absolute vorticity is not mixed across the equator but separately by the Rossby wave lobes on either side of the equator.

If the equatorial superrotating jet corresponding to the end state with hemispherically homogenized absolute vorticity has half-width $L_{s}$, and if the absolute vorticity at the zero of the zonal velocity at the distance $L_{s}$ 

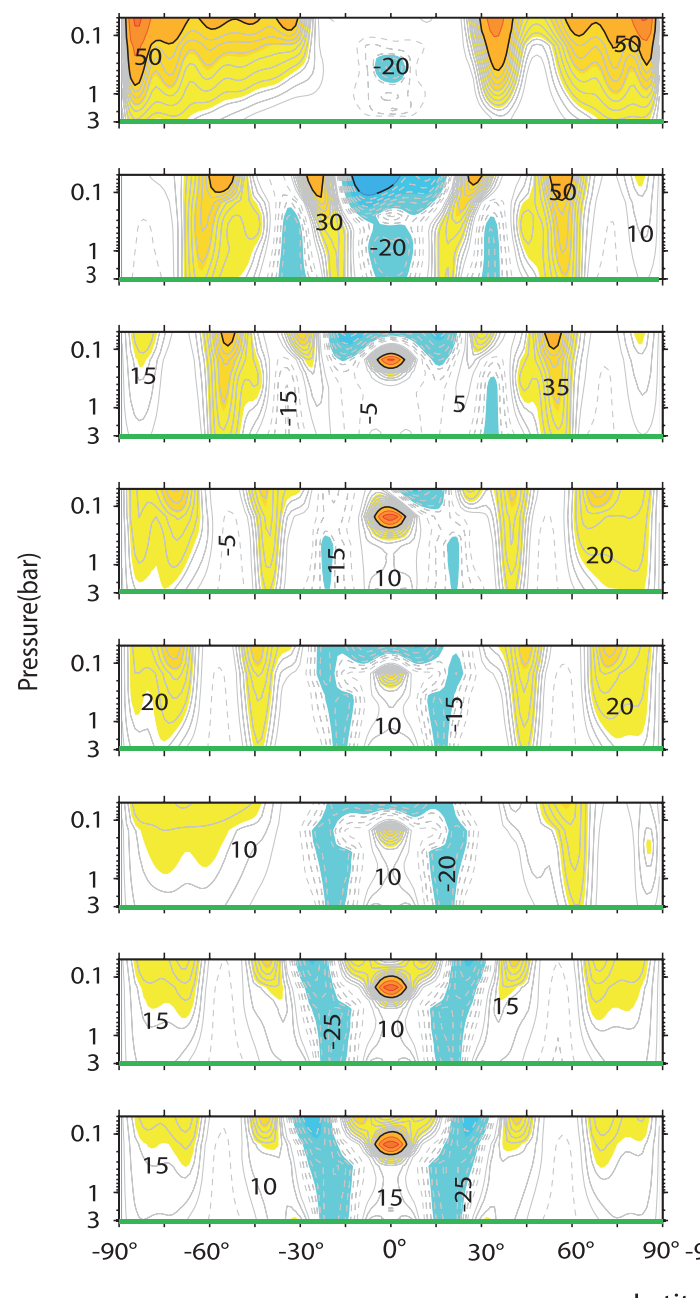

Latitude

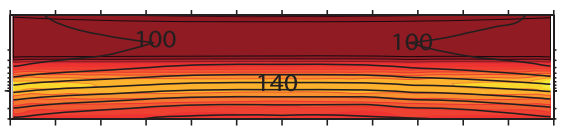

$0.0 \mathrm{~W} \mathrm{~m}^{-2}$

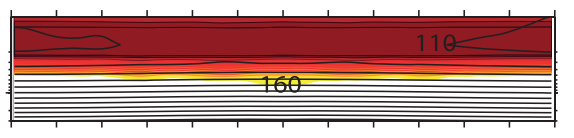

$5.0 \mathrm{~W} \mathrm{~m}^{-2}$

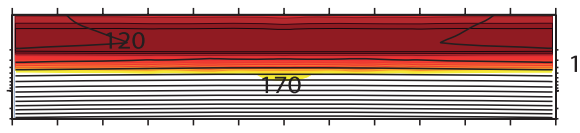

$10.0 \mathrm{~W} \mathrm{~m}^{-2}$
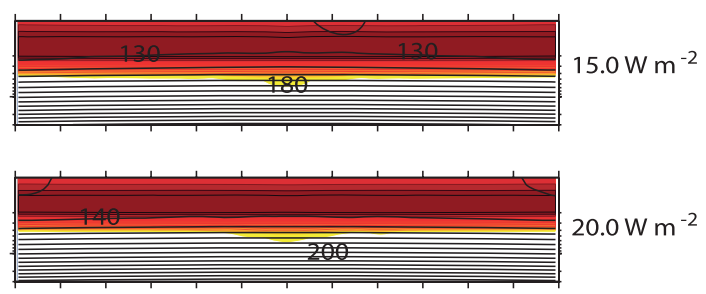

$20.0 \mathrm{~W} \mathrm{~m}^{-2}$

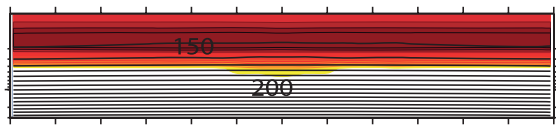

$25.0 \mathrm{~W} \mathrm{~m}^{-2}$

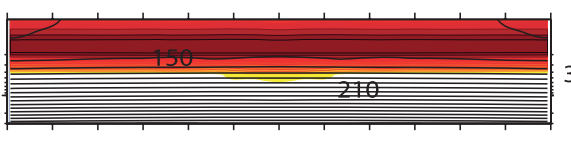

$30.0 \mathrm{~W} \mathrm{~m}^{-2}$
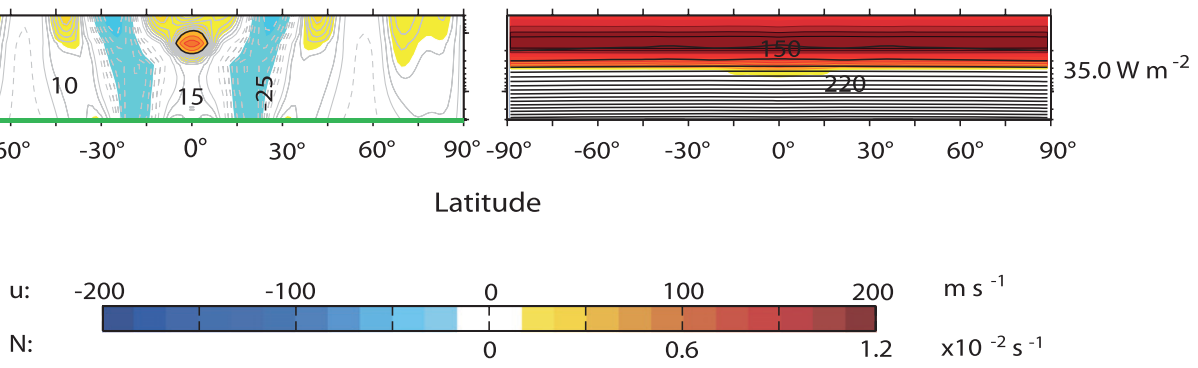

FIG. 8. Simulations with weak insolation gradient $\Delta s=0.4$ and with bottom drag with constant coefficient at all latitudes (case B). Contour intervals and plotting conventions as in Figs. 2 and 7.

away from the equator is approximately $\beta L_{s}$, as sketched in Fig. 10, the mean relative vorticity between the equator and a distance $L_{s}$ away from it is $\beta L_{s} / 2$. The speed of the jet at the equator then follows by integration of the relative vorticity from the zero at the distance $L_{s}$ toward the equator and is (Rhines 1994; SL09)

$$
U_{\max }=\frac{\beta L_{s}^{2}}{2}
$$

This speed represents an upper bound on the equatorial velocity for a jet of half-width $L_{s}$, as any deviation from absolute vorticity homogenization in each hemisphere implies a weaker equatorial jet. Note that although the mean vorticity (and so the meridional derivative of the mean zonal velocity) jumps at the equator in the homogenized end state, the mean zonal velocity there is continuous. Figure 10 shows that even in a simulation with strong intrinsic heat flux and a strong equatorial jet (cf. Fig. 2), the end state of hemispherically homogenized vorticity is not attained, but absolute vorticity is approximately homogenized in the flanks of the jet. However, although the bound (6) is not attained, the strength of the superrotating jets may still scale the way the bound indicates.

\section{b. Jet strength}

Figure 11 shows that the actual strength of superrotating jets in our simulations indeed scales with the upper 

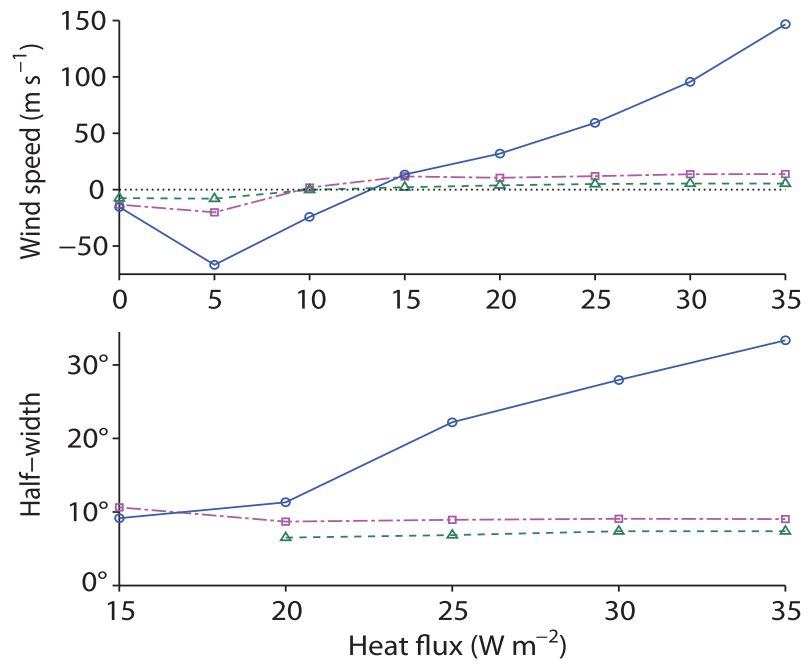

FIG. 9. Vertically averaged strength and width of the equatorial jets in the simulations with $\Delta s=0.4$ and with different bottom drag formulations. (a) Equatorial zonal velocity as a function of the intrinsic heat flux, averaged from $-1^{\circ}$ to $1^{\circ}$ latitude meridionally and from $\sigma=0.1$ ( 0.3 bar) to $\sigma=0.8$ (2.4 bar) vertically. (b) Halfwidth of the equatorial superrotating jet as a function of the intrinsic heat flux. (Only jets for which superrotation occupies the entire troposphere are shown). Blue circles with solid lines for no drag in the equatorial region (case A, as in Fig. 5); magenta squares with dashed-dotted lines for weak drag with latitudinally constant coefficient (case B); green triangles for stronger drag with latitudinally constant coefficient (case C).

bound (6). The vertically averaged zonal wind speed in the equatorial region is consistently about a quarter of the upper bound $U_{\max }$. (The exact strength of the superrotating equatorial jets depends on the subgrid-scale dissipation in the GCM, so we do not expect this onequarter fraction to be universal.) In particular, this scaling relation shows that the strength of the jets increases quadratically with their width, which is also roughly true for Jupiter and Saturn: Saturn's superrotating equatorial jet at the level of observed clouds is about twice as wide and up to 4 times as strong as Jupiter's (see, e.g., Fig. 1 in LS10).

\section{c. Jet width}

The relation between jet strength and width raises the question of how the width of the superrotating equatorial jets is controlled. Given that the jets come about through convectively generated equatorial Rossby waves, and if the dissipation leading to angular momentum transport primarily occurs in their lobes, the width of the jets can be expected to scale with the meridional scale of the waves (Fig. 3a), the equatorial Rossby radius

$$
L_{\beta} \sim\left(\frac{c}{\beta}\right)^{1 / 2} .
$$

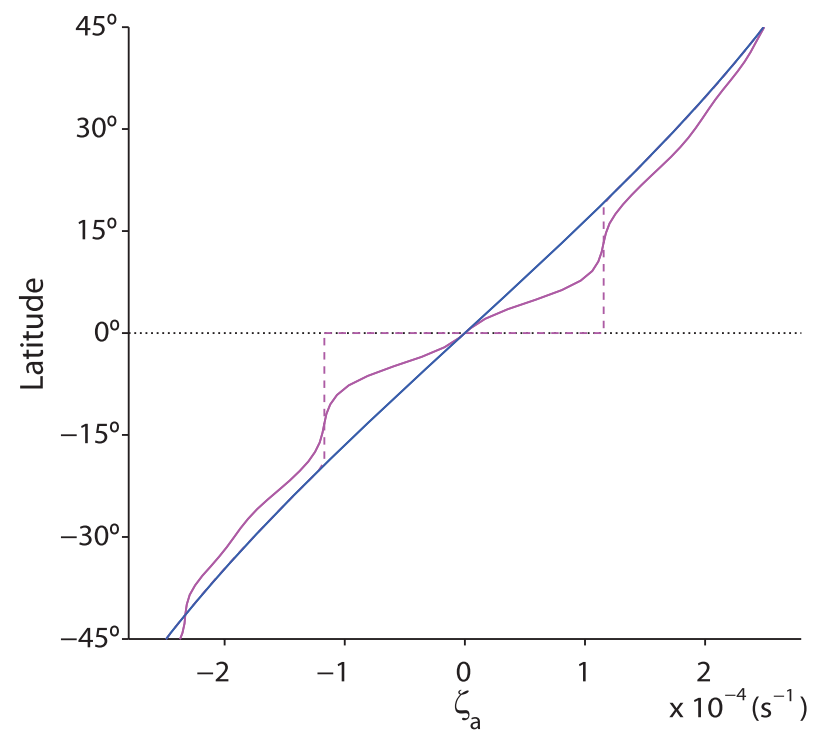

FIG. 10. Latitudinal absolute vorticity profiles. The blue line shows the planetary vorticity $f=2 \Omega \sin \phi$; the dashed magenta line shows the end state of homogenized absolute vorticity in each hemisphere; and the solid magenta line shows the absolute vorticity at 2 bar in the simulation with an intrinsic heat flux of $35.0 \mathrm{~W} \mathrm{~m}^{-2}$, $\Delta s=0.4$, and no drag in the equatorial region (case A).

To test whether this is the case, we determine the gravity wave speed $c$ entering the equatorial Rossby radius from the static stability of the atmosphere, estimating it as $c=\int_{p_{s}}^{p_{t}} N_{p} d p$, where $N_{p}$ is the static stability measure $N_{p}=-(\bar{\rho} \bar{\theta})^{-1} \frac{\overline{\partial_{p}} \theta}{p}, p_{s}$ and $p_{t}$ indicate the pressure range of the integration, and the notation is otherwise standard (e.g., Schneider and Walker 2006). Here we take $p_{s}=2.4$ bar (in the troposphere but above the boundary layer of the model) and $p_{t}=0.75$ bar (below the tropopause). The precise choice of these levels does not substantially affect our results.

Figure 12 shows that the half-width of the superrotating equatorial jets in many simulations indeed scales roughly with the equatorial Rossby radius. Deviations from this scaling occur principally in the simulations with drag in the equatorial region, particularly with strong drag (case C). In these simulations, the superrotating jets are weak (see Fig. 8) or, in case C, extremely weak (see Fig. 9). It is plausible that in these cases, the jet width is not set by the convectively generated Rossby waves alone, but that mean meridional circulations interacting with extratropical baroclinic eddies also play a role, as in Earth's atmosphere (e.g., Held 2000; Walker and Schneider 2006; Korty and Schneider 2008), leading to deviations from the scaling. Nonetheless, the scaling appears roughly adequate at least for the simulations that exhibit strong equatorial superrotation.

Combining the facts that the strength of at least the strongly superrotating jets scales with the upper bound 


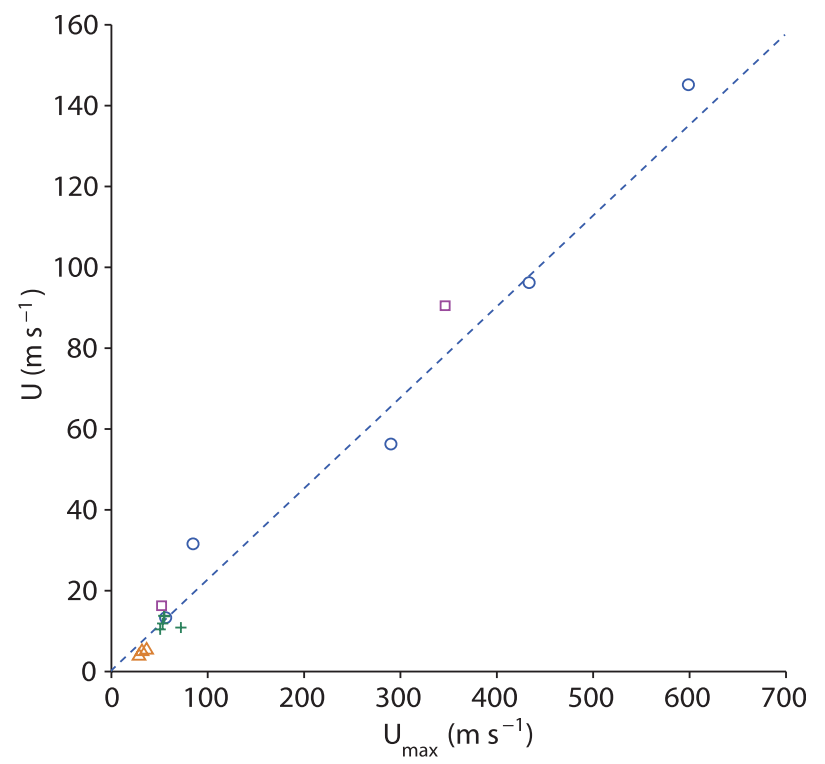

FIG. 11. Zonal velocity $U$ of superrotating jets, averaged from $-1^{\circ}$ to $1^{\circ}$ latitude meridionally and from $\sigma=0.1$ ( 0.3 bar) to $\sigma=0.8$ (2.4 bar) vertically, versus the upper bound $U_{\max }=\beta L_{s}^{2} / 2$. (Only jets for which superrotation occupies the entire troposphere are shown.) Here, the half-width $L_{s}$ of the jets is determined as the distance from the equator of the first zero of the vertically averaged zonal velocity. The dashed line represents $U=0.225 U_{\max }$. Blue circles for $\Delta s=0.4$ and no drag in the equatorial region (case $\mathrm{A}$ ); magenta squares for $\Delta s=0.7$ and no drag in the equatorial region (case A); green crosses for $\Delta s=0.4$ and constant weak drag (case B); orange triangles for $\Delta s=0.4$ and constant strong drag (case C).

(6) and that their width scales with the equatorial Rossby radius, we obtain that the speed of the jets scales with the gravity wave speed:

$$
U \sim c .
$$

The gravity wave speed and thus the static stability of the equatorial troposphere thus emerge as central quantities determining characteristics of the equatorial superrotating jets. The static stability is controlled by the flow itself and is not externally given, so these scaling relations do not provide a closed theory. To obtain a closed theory, it is necessary to relate the static stability to other flow characteristics. The static stability in the equatorial troposphere generally increases as the intrinsic heat flux increases beyond the threshold at which superrotation occurs (Fig. 2). One may suppose that convection in the equatorial region leads to homogenization of entropy along angular momentum surfaces, which are deformed away from the vertical in the equatorial region through the superrotating jet. Thus, convection would establish a state that is neutral with respect to symmetric instabilities (e.g., Thorpe and Rotunno 1989). However, so far we have not been able to obtain a quantitatively adequate fit of such or similar ideas to the simulation results.

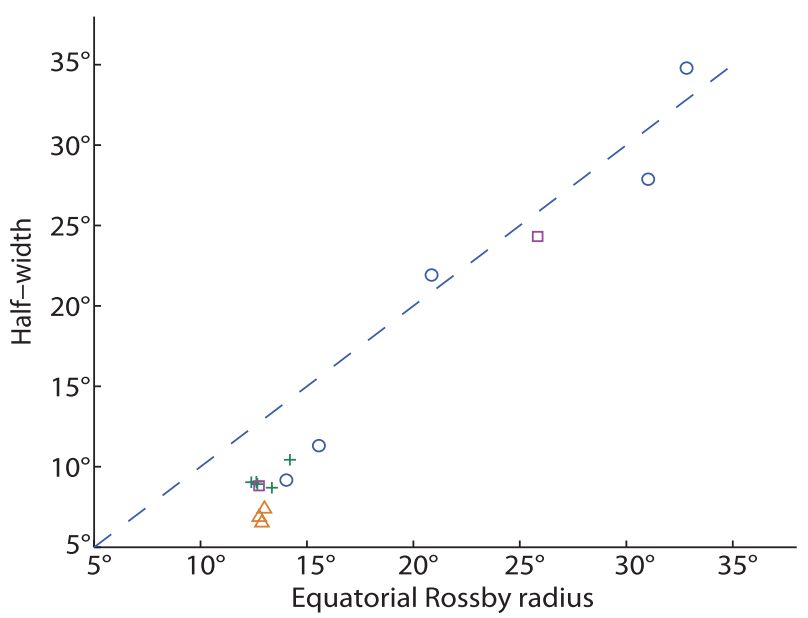

FIG. 12. Equatorial Rossby radius $L_{\beta}$ vs the half-width $L_{s}$ of the superrotating equatorial jets. (Only jets for which superrotation occupies the entire troposphere are shown.) The equatorial Rossby radius is estimated as $L_{\beta}=4.0(c / \beta)^{1 / 2}$, with gravity wave speed $c$ estimated as described in the text. The dashed line represents $L_{\beta}=L_{s}$. Plotting symbols as in Fig. 11.

\section{Conclusions, discussion, and open questions}

We have carried out a systematic simulation study with an idealized GCM to investigate how heating from below, baroclinicity, and bottom drag determine when equatorial superrotation occurs in rapidly rotating atmospheres and how the strength and width of superrotating equatorial jets are controlled. In general, as the heating at the bottom boundary strengthens, a transition to equatorial superrotation occurs at a threshold heat flux. This transition appears to be gradual as a function of heat flux, unlike the abrupt transitions seen in quasigeostrophic two-layer models with a stationary heat source (Suarez and Duffy 1992; Saravanan 1993). The superrotating equatorial jets continue to strengthen and widen as the heat flux increases beyond the superrotation threshold. However, the threshold depends on the baroclinicity of the flow and on the bottom drag (and on other dissipation mechanisms such as subgrid-scale dissipation in a GCM). The following general inferences can be drawn:

(i) The greater the off-equatorial baroclinicity, the greater the threshold heat flux at which superrotation occurs and the weaker and narrower the resulting superrotating equatorial jets. Angular momentum transport by extratropical baroclinic eddies out of low latitudes competes with angular momentum transport by convectively generated Rossby waves into the equatorial region. For superrotation to occur, stronger baroclinic eddy fluxes hence require stronger convectively generated Rossby waves and so stronger heat fluxes. 
(ii) The stronger the bottom drag in the equatorial region, the greater the threshold heat flux at which superrotation occurs and the weaker and narrower the resulting superrotating equatorial jets. Equatorial bottom drag damps both the mean zonal flow and convectively generated Rossby waves, weakening the angular momentum transport into the equatorial upper troposphere and inhibiting a transition to superrotation.

(iii) The strength of superrotating equatorial jets scales approximately with the square of their width. When they are sufficiently strong, their width, in turn, scales with the equatorial Rossby radius. It thus depends on the thermal stratification of the equatorial atmosphere, which is controlled by the flow in a manner that remains unclear.

The results thus provide some general, if only qualitative, rules when superrotation in planetary atmospheres may be expected. For example, as already discussed in LS10, superrotation occurs on Jupiter and Saturn but not on Uranus and Neptune, apparently because the intrinsic heat fluxes on Uranus and Neptune are too weak (they are essentially zero on Uranus) to overcome the offequatorial baroclinicity, which in itself leads to equatorial subrotation. Similarly, the transition to superrotation that Caballero and Huber (2010) observed in an Earth climate model under extreme global warming may arise because, on the one hand, baroclinicity in the warm climate is reduced and, on the other hand, convective heating fluctuations may intensify, leading to an increased importance of convectively generated Rossby waves in the equatorial angular momentum balance. Or the transitions to superrotation in Earth-like models as the rotation rate is reduced (e.g., Walker and Schneider 2006) or as the planetary radius is reduced (e.g., Mitchell and Vallis 2010) may likewise arise, as suggested by Mitchell and Vallis (2010), because of shifts in the importance for the equatorial angular momentum balance of extratropical baroclinic eddy fluxes relative to equatorial eddy fluxes (which do not have to be convectively generated). ${ }^{1}$

What remains unsatisfying about these statements is that they are not quantitative; that is, we do not have a

\footnotetext{
${ }^{1}$ Mitchell and Vallis (2010) emphasize a transition to superrotation that occurs when the importance of extratropical baroclinic eddy fluxes in the angular momentum balance diminishes as the thermal Rossby number becomes order one and larger. However, our results and those of Caballero and Huber (2010) show that a transition to superrotation in terrestrial atmospheres (i.e., shallow atmospheres interacting with a surface) can occur even when the thermal Rossby number is small [it is $O\left(10^{-2}\right)$ in our simulations], provided the external heating of the surface is sufficiently strong and surface drag is sufficiently weak.
}

quantitative theory that gives, for example, the threshold heat flux for superrotation as a function of baroclinicity and drag parameters. Because our results show that such a theory must involve a theory of atmospheric macroturbulence and of the effects of drag on equatorial dynamics, it may be difficult to develop. It also remains to develop a theory of the thermal stratification in the equatorial troposphere, which determines the strength and width of the superrotating equatorial jets.

Acknowledgments. This work was supported by a David and Lucile Packard Fellowship and by the NASA Outer Planets Research Program (Grant NNX10AQ05G). The GCM is based on the Flexible Modeling System of the Geophysical Fluid Dynamics Laboratory. The simulations were performed on Caltechs Division of Geological and Planetary Sciences Dell cluster.

\section{APPENDIX}

\section{Sensitivity of Results to Convection Scheme}

The quasi-equilibrium convection scheme used in the GCM simulations in the main part of this paper relaxes temperature profiles toward a convective profile with adiabatic lapse rate $\Gamma=g / c_{p}$ on a time scale $\tau_{c}=6 \mathrm{~h}$. This is roughly the time it takes a gravity wave with speed $c$ to traverse the extratropical Rossby radius $L_{x}=c / f$ (i.e., an extratropical inertial time $\tau_{c} \sim f^{-1}$ ). The convection scheme does not transport momentum. Here we investigate the effect of the convective relaxation time and of convective momentum transport on the largescale circulations. We present results from simulations with convective relaxation times of $0.6 \mathrm{~h}$ (close to the instantaneous convective adjustment limit) and $60 \mathrm{~h}$ and of a simulation with a convection scheme that also homogenizes momentum where convection occurs.

Figure A1 shows the results. The simulation considered has an intrinsic heat flux of $25.0 \mathrm{~W} \mathrm{~m}^{-2}$ and $\Delta s=$ 0.4 with no drag in the equatorial region, which in the standard case leads to equatorial superrotation. Compared with the standard case (Fig. A1a), reducing the convective relaxation time by a factor of 10 (Fig. A1b) leads only to small changes in the structure and speed of the jets and in the thermal stratification. Our results appear to be relatively insensitive to reductions of the convective relaxation time, likely because the convective relaxation times chosen are short compared with typical wave times scales. However, increasing the convective relaxation time by a factor of 10 (Fig. A1c) leads to a stronger and wider superrotating equatorial jet. Consistent with the stronger and wider equatorial jet and with the scaling arguments in section 6 , the thermal stratification 


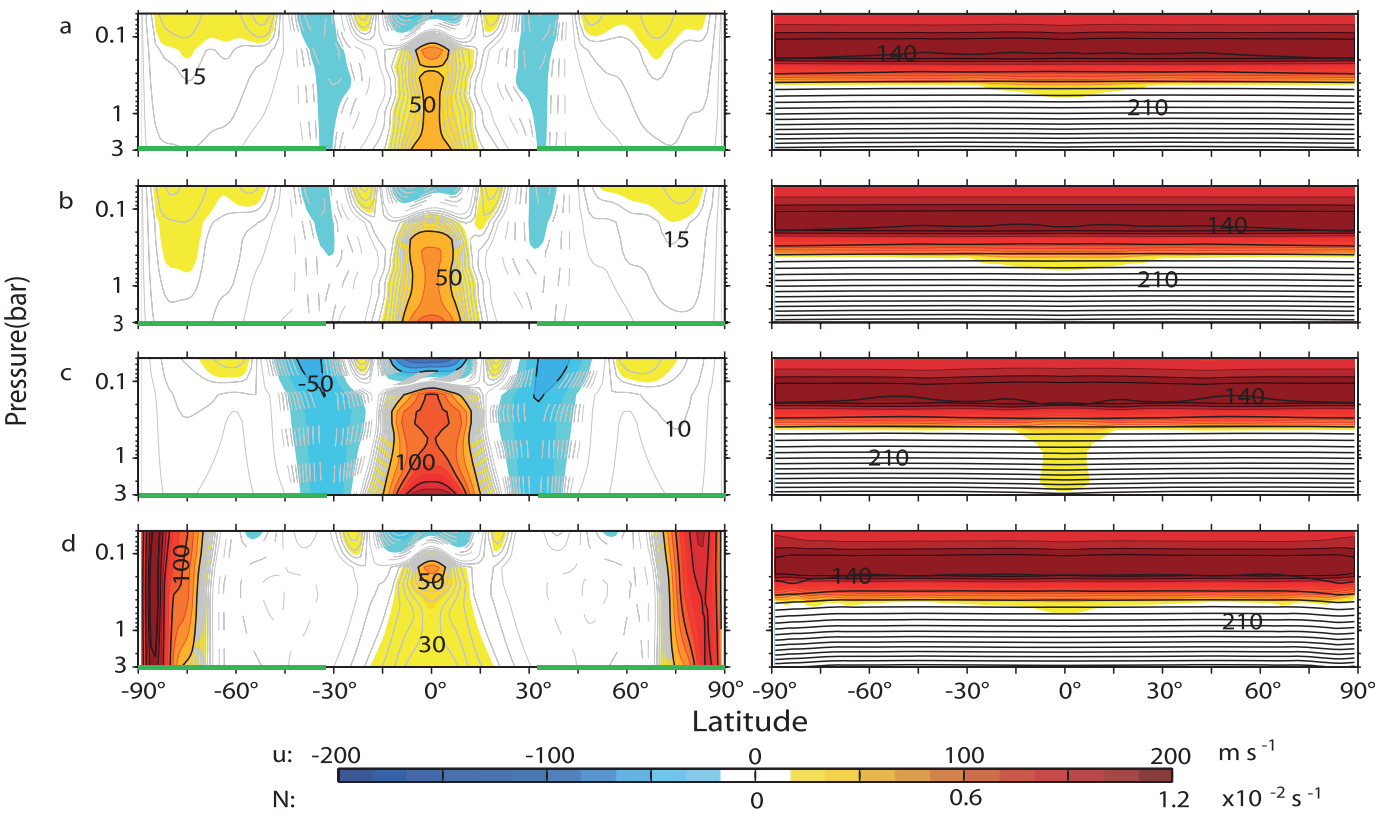

FIG. A1. Simulations with $25.0 \mathrm{~W} \mathrm{~m}^{-2}, \Delta s=0.4$, and no drag in the equatorial region, with different parameterizations of convection: (a) $\tau_{c}=6 \mathrm{~h}$ (standard case); (b) $\tau_{c}=0.6 \mathrm{~h}$; (c) $\tau_{c}=60 \mathrm{~h}$; (d) $\tau_{c}=6 \mathrm{~h}$ and horizontal momentum homogenization on the same time scale below the zero buoyancy level. Note that (a) reproduces the corresponding panel in Fig. 2 for ease of comparison. Contour intervals and plotting conventions are as in Figs. 2 and 7.

is statically more stable near the equator. The results in section 6 suggest that the less efficient convection allows the large-scale flow to establish a statically more stable thermal stratification, implying an increased gravity wave speed, and that the stronger and wider equatorial jet is a consequence.

Because turbulent Prandtl numbers in dry convection generally are substantially greater than zero, neglect of momentum transport by convection may be a serious omission that may affect our results. But if horizontal momentum over the convective part of the column is relaxed toward the vertical average on the same time scale $\tau_{c}=6 \mathrm{~h}$ over which temperatures are relaxed, the equatorial superrotating jet is not substantially changed, although it becomes weaker and, interestingly, develops larger vertical shear (Fig. A1d). However, there are substantial changes in the extratropics, with strong polar vortices with deep barotropic structures developing. Some of the changes in the equatorial region may arise because the convective momentum mixing allows a strong mean meridional circulation to develop, which reaches the model surface and whose upper and lower branches are coupled through convective momentum transport. Without parameterized convective momentum transport and without drag in the equatorial region, the equatorial overturning cells (see Fig. 4 for an example) are weaker (by a factor of 3.0 in the case considered here) because momentum transport out of the lower boundary layer is required to close the circulations at the bottom (e.g., LS10). Without parameterized convective momentum fluxes and without drag, this has to be supplied by momentum fluxes on the grid scale of the model or parameterized subgrid-scale fluxes.

In summary, although a more exhaustive exploration of convective effects and in particular convective momentum transport would be desirable, we feel confident that the main results of this paper are not substantially affected by convective relaxation times (as long as they are sufficiently short) or convective momentum transport.

\section{REFERENCES}

Caballero, R., and M. Huber, 2010: Spontaneous transition to superrotation in warm climates simulated by CAM3. Geophys. Res. Lett., 37, L11701, doi:10.1029/2010GL043468.

Charney, J. G., 1963: A note on large-scale motions in the tropics. J. Atmos. Sci., 20, 607-609.

, 1969: A further note on large-scale motions in the tropics. J. Atmos. Sci., 26, 182-185.

Held, I. M., 1999: Equatorial superrotation in Earth-like atmospheric models. Bernhard Haurwitz Memorial Lecture, American Meteorological Society. [Available online at at http:// www.gfdl.noaa.gov/cms-filesystem-action/user_files/ih/lectures/ super.pdf.]

, 2000: The general circulation of the atmosphere. Proc. Program in Geophysical Fluid Dynamics, Woods Hole Oceanographic 
Institution, Woods Hole, MA. [Available online at http:// www.gfdl.noaa.gov/cms-filesystem-action/user_files/ih/lectures/ woods_hole.pdf.]

- and M. J. Suarez, 1994: A proposal for the intercomparison of the dynamical cores of atmospheric general circulation models. Bull. Amer. Meteor. Soc., 75, 1825-1830.

Korty, R. L., and T. Schneider, 2008: Extent of Hadley circulations in dry atmospheres. Geophys. Res. Lett., 35, L23803, doi:10.1029/2008GL035847.

Kostiuk, T., K. E. Fast, T. A. Livengood, T. Hewagama, J. J. Goldstein, F. Espenak, and D. Buhl, 2001: Direct measurement of winds on Titan. Geophys. Res. Lett., 28, 2361-2364.

Liu, J., and T. Schneider, 2010: Mechanisms of jet formation on the giant planets. J. Atmos. Sci., 67, 3652-3672.

Lodders, K., and B. Fegley Jr., 1998: The Planetary Scientist's Companion. Oxford University Press, $400 \mathrm{pp}$.

Mitchell, J. L., and G. K. Vallis, 2010: The transition to superrotation in terrestrial atmospheres. J. Geophys. Res., 115, E12008, doi:10.1029/2010JE003587.

O'Gorman, P. A., and T. Schneider, 2008: Weather-layer dynamics of baroclinic eddies and multiple jets in an idealized general circulation model. J. Atmos. Sci., 65, 524-535.

Peixoto, J. P., and A. H. Oort, 1992: Physics of Climate. American Institute of Physics, $555 \mathrm{pp}$.

Porco, C. C., and Coauthors, 2003: Cassini imaging of Jupiter's atmosphere, satellites, and rings. Science, 299, 1541-1547.

Randel, W. J., and I. M. Held, 1991: Phase speed spectra of transient eddy fluxes and critical layer absorption. J. Atmos. Sci., 48, 688-697.

Rhines, P. B., 1994: Jets. Chaos, 4, 313-339.

Sanchez-Lavega, A., R. Hueso, and S. Perez-Hoyos, 2007: The three-dimensional structure of Saturn's equatorial jet at cloud level. Icarus, 187, 510-519.

Saravanan, R., 1993: Equatorial superrotation and maintenance of the general circulation in two-level models. J. Atmos. Sci., 50, 1211-1227.
Sardeshmukh, P. D., and B. J. Hoskins, 1988: The generation of global rotational flow by steady idealized tropical divergence. J. Atmos. Sci., 45, 1228-1251.

Schneider, T., 2006: The general circulation of the atmosphere. Annu. Rev. Earth Planet. Sci., 34, 655-688.

— macroturbulence into critical states of weak nonlinear eddyeddy interactions. J. Atmos. Sci., 63, 1569-1586.

_ turbulence in dry atmospheres. J. Atmos. Sci., 65, 2153-2173.

rotation on Jupiter. J. Atmos. Sci., 66, 579-601.

Schubert, G., 1983: General circulation and dynamical state of Venus. Venus, D. M. Hunten, Ed., University of Arizona Press, 681-765.

Shell, K. M., and I. M. Held, 2004: Abrupt transition to strong superrotation in an axisymmetric model of the upper troposphere. J. Atmos. Sci., 61, 2928-2935.

Smith, K. S., G. Boccaletti, C. C. Henning, I. Marinov, C. Y. Tam, I. M. Held, and G. K. Vallis, 2002: Turbulent diffusion in the geostrophic inverse cascade. J. Fluid Mech., 469, 13-48.

Sobel, A. H., J. Nilsson, and L. M. Polvani, 2001: The weak temperature gradient approximation and balanced tropical moisture waves. J. Atmos. Sci., 58, 3650-3665.

Sromovsky, L. A., A. D. Collard, P. M. Fry, G. S. Orton, M. T. Lemmon, M. G. Tomasko, and R. S. Freedman, 1998: Galileo probe measurements of thermal and solar radiation fluxes in the Jovian atmosphere. J. Geophys. Res., 103, 22 929-22 977.

Suarez, M. J., and D. G. Duffy, 1992: Terrestrial superrotation: A bifurcation of the general circulation. J. Atmos. Sci., 49, 15411554.

Thorpe, A. J., and R. Rotunno, 1989: Nonlinear aspects of symmetric instability. J. Atmos. Sci., 46, 1285-1299.

Walker, C. C., and T. Schneider, 2006: Eddy influences on Hadley circulations: Simulations with an idealized GCM. J. Atmos. Sci., 63, 3333-3350. 